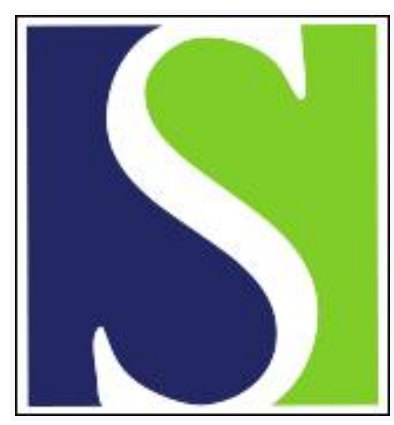

Scand J Work Environ Health 1997;23(2):149-151

https://doi.org/10.5271/sjweh.192

Issue date: Apr 1997

Asthma in patients with farmer's lung during a five-year follow-up

by Kokkarinen Jl, Tukiainen HO, Terho EO

Key terms: agricultural worker; extrinsic allergic alveolitis; occupational disease

This article in PubMed: www.ncbi.nlm.nih.gov/pubmed/9167238

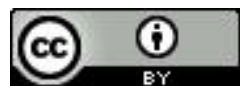




\title{
Asthma in patients with farmer's lung during a five-year follow-up
}

\author{
by Jouko I Kokkarinen, MD, ${ }^{1}$ Hannu O Tukiainen, MD, ${ }^{1}$ Erkki O Terho, MD ${ }^{2}$
}

\begin{abstract}
Kokkarinen JI, Tukiainen HO, Terho EO. Asthma in patients with farmer's lung during a five-year follow-up. Scand $J$ Work Environ Health 1997;23(2):149-51.

Objectives This study assessed the incidence of asthma in patients with farmer's lung.

Methods The study population consisted of 1031 patients who had occupational farmer's lung diagnosed from 1983 to 1988. The cases of farmer's lung were registered in the nationwide Register of Occupational Diseases by the Finnish Institute of Occupational Health, and all the patients in the study had received compensation from the insurance company for an occupational disease. The diagnosis of asthma was based on entitlement to a special refund for asthma medication costs from the national sickness insurance program.

Results Seventy-four cases of asthma were found among farmers with farmer's lung up to the end of the 5 years following the year of the diagnosis for farmer's lung. The prevalence of asthma was $1 \%$ in the year preceding the diagnosis. In the year of the diagnosis and during the 5 years of follow-up, asthma was diagnosed in an additional $6 \%$ of the patients.

Conclusions The results of this study confirm previous findings of a low prevalence of asthma before the onset of farmer's lung in small groups of patients. However, the results also indicate that the incidence of asthma increases during the first years after an episode of farmers lung. For example, in the present study, asthma was apparent in $7 \%$ of the patients 5 years after the diagnosis of farmer's lung.
\end{abstract}

Key terms agricultural workers, extrinsic allergic alveolitis, occupational diseases.

Asthma preceding the onset of farmer's lung has seldom been reported (1). However, some farmer's lung patients may also show an obstructive pattern in inhalation challenge with moldy hay or other offending antigens $(1,2)$. In a few studies asthma has also been reported in $7 \%$ to $18 \%$ of patients during follow-up after an episode of farmer's lung (3-5).

To assess the association between asthma and farmer's lung in a larger group of patients, a total of 1031 farmer's lung cases diagnosed between 1983 and 1988 were identified from the Finnish Register of Occupational Diseases. The data of the patients were aggregated to the data on entitlement to a special refund for asthma medication costs from the national sickness insurance program, as registered by the Social Insurance Institution.

\section{Material and methods}

Farmer's lung cases were identified from the nationwide Register of Occupational Diseases that is kept by the Finnish Institute of Occupational Health in Helsinki. In Finland, cases of farmer's lung are mainly diagnosed by pulmonary physicians in a hospital setting. The diagnostic criteria presented for farmer's lung by Terho (6) are widely used in the diagnosis. The insurance companies also follow these criteria in assessing possible compensation for farmer's lung. Cases in which the diagnosis of farmer's lung was made from 1983 to 1988 were included in this study. In addition, the cases were only included if compensation for farmer's lung as an occupational disease had been reimbursed by the insurance company. Thus the study population consisted of 1031 farmer's lung cases. The number of the cases diagnosed each year was as follows: 64 in 1983, 69 in 1984, 156 in 1985, 218 in 1986, 216 in 1987, and 308 in 1988. There were 651 $(63 \%)$ female patients and $380(37 \%)$ male patients. The mean age of the patients at the time of the diagnosis was 48 (range $18-65$ ) years.

Patients with asthma were identified from the register of the Social Insurance Institution of Finland. In Finland, the cost of medication prescribed by a physician is refunded through the national sickness insurance program. The basic refund has been $50 \%$ of medication costs in excess of a fixed deductible amount. In addition,

1 Department of Pulmonary Diseases, Kuopio University Hospital, Kuopio, Finland.

2 Department of Pulmonary Diseases and Clinical Allergology, University of Turku, Turku, Finland.

Reprint requests to: Dr Jouko Kokkarinen, Department of Pulmonary Diseases, Kuopio University Hospital, FIN-70210 Kuopio, Finland. 
certain serious and long-term diseases, including bronchial asthma, are entitled to a special refund of $80 \%$ to $100 \%$. The special refund for asthma medication has been as follows: $100 \%$ from 1970 to $1985,90 \%$ from 1986 to 1991 , and $80 \%$ from 1992 to 1993.

To receive the special refund, the patient must have chronic asthma, diagnosed usually by a pulmonary physician. The criteria for asthma are compatible clinical symptoms and demonstration of reversible bronchial obstruction [(a) improvement in peak expiration flow (PEF) by at least $15 \%$ and $50 \mathrm{1} / \mathrm{min}$ or in forced expiratory volume in $1 \mathrm{~s}\left(\mathrm{FEV}_{1.0}\right)$ by at least $15 \%$ and $0.151 \mathrm{in} \mathrm{a}$ bronchodilator test or (b) fall in PEF of at least $15 \%$ and $501 / \mathrm{min}$ or in $\mathrm{FEV}_{1.0}$ of at least $15 \%$ and 0.151 in a clinical exercise test or (c) at least $20 \%$ and $50 \mathrm{l} / \mathrm{min}$ diurnal variation in peak flow monitoring]. Entitlement to a special refund can be provided at the time of the diagnosis of asthma in clear cases or up to 12 months thereafter once the chronic nature of the disease is ascertained. Permanent or temporary entitlement to a special refund is provided and registered by the Social Insurance Institution on the basis of the physician's statement about the disease. Our search of the register included a 5-year follow-up after the year of diagnosis of farmer's lung.

The prevalence of asthma among farmer's lung patients was compared with the statistics of the Social Insurance Institution concerning data on entitlement to a special refund for asthma medication costs in the entire population. The median year for the diagnosis of farmen's lung was 1987 , and therefore the situation preceding the diagnosis of farmer's lung was compared with the statistical data from 31 December 1986 and that at the 5year follow-up with the data from 31 December 1992.

\section{Results}

Seventy-four $(7.2 \%)$ of the farmer's lung patients were entitled to a special refund for asthma medication during

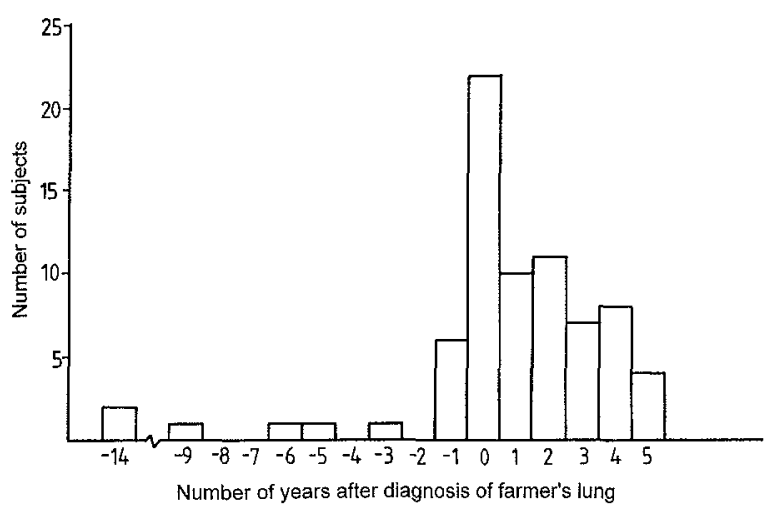

Figure 1. Diagnosis of asthma based on entitlement to receive a special refund for asthma medication costs in regard to the year of the diagnosis of farmer's lung. the 5 years after the year of diagnosis for farmer's lung (figure 1). Forty-three of the 74 patients were entitled to a special refund within 3 years of the year of the farmer's lung diagnosis. The age (mean of 49 years at the time of the diagnosis of farmer's lung) and gender (42 women, 32 men) of the patients with a diagnosis of asthma did not differ from that of farmer's lung patients without asthma [age: $\mathrm{P}=0.28$ (analysis of variance), gender: $\mathrm{P}=0.24$, chi-square $]$.

Twelve of the patients (1.2\%) had asthma already in the year preceding the diagnosis of farmer's lung, and half of them were entitled to a special refund for asthma medication the preceding year. For the entire Finnish population in the age group 45 to 54 years the comparable figure was $2.0 \%$ (also $2.0 \%$ after adjustment for gender) on 31 December 1986.

Entitlement to a special refund for asthma medication was provided permanently for 70 patients and temporarily for 4 . For 2 of the latter 4 the entitlement was no longer in force at the time of the 5-year follow-up. Thus the prevalence of asthma at the 5-year follow-up was $7.0 \%$. For the entire Finnish population in the age group 50 to 59 years, the corresponding figure was $3.5 \%$ (3.6\% after adjustment for gender) in 31 December 1992.

\section{Discussion}

We found a clustering of asthma especially within the first 3 years after the year of diagnosis of farmer's lung. In our previous study (3), 5 out of 7 cases of asthma in 101 farmer's lung patients were diagnosed within 2 years after the diagnosis of farmer's lung.

The percentage of patients entitled to a special refund for asthma medication in the year preceding the diagnosis of farmer's lung was low compared with that of the general population of the same age. This finding agrees with the reported low prevalence of asthma preceding the occurrence of farmer's lung. In 2 Finnish follow-up studies examining 86 and 101 farmer's lung patients, no patient had asthma at the time of the diagnosis of farmer's lung $(3,7)$. Pepys reported previous asthma in 4 of a total of 205 patients (2.0\%) with farmer's lung (1).

For $2.1 \%$ of the patients, the entitlement to a special refund for asthma medication was awarded in the same year as the diagnosis of farmer's lung. Karr and his coworkers have reported a case with concomitant development of asthma and farmer's lung (8).

Five years after the diagnosis of farmer's lung, the prevalence of asthma was $7.0 \%$. This rate is higher than the $3.6 \%$ of the reference population. Our results agree with those of Pepys, who stated that asthma may develop in about $10 \%$ of subjects some time after the onset of farmer's lung (4). In our previous study of 101 farmer's lung patients, the prevalence of asthma was also $7 \%$ at the 5-year follow-up (3). In a 10-year follow-up study of 
27 patients, asthma was reported for 5 patients (18\%) (5).

In a large epidemiologic study of Finnish farmers in 1979 , the prevalence of asthma was $3.3 \%$ (9). In the register of the Social Insurance Institution for entitlement to a special refund for medication, occupation is not recorded, and therefore data on the proportion of farmers receiving a refund for asthma medication was not available. Asthma in farmers may be more common than in the general population; therefore no current data are available on whether the prevalence of asthma in our study $(7 \%)$ is of the same order or even higher than that for farmers without farmer's lung. Nonetheless, the cases of asthma in our study were clustered into the first few years after the episode of farmer's lung, and therefore an association between farmer's lung and the onset of asthma is suggested.

In the acute stage of farmer's lung, bronchial hyperreactivity to histamine has been demonstrated in $27 \%$ of patients (10). The bronchial hyperreactivity was transient in the majority of the patients. Receptors for the Fc portion of immunoglobulin $\mathrm{E}$ ( $\mathrm{IgE}$ ) have been detected on the surface of alveolar macrophages in both farmer's lung and bird fancier's lung (11). It has therefore been suggested that IgE-related immune reactions might be involved in allergic alveolitis (11). These phenomena may be related to the onset of asthma for some patients after an episode of farmer's lung.

We recorded asthma on the basis of data on entitlement to a special refund for medication costs. Milder cases of asthma and cases with only occasional symptoms may not have been included. Thus the true prevalence of asthma might be higher. Because of the major difference between the basic refund and special refund, patients with asthma and regular asthma medication are likely to be registered. Entitlement to a special refund for asthma medication can be obtained up to a year after the diagnosis of asthma, if the chronicity of the disease is ascertained. This time span may lead to some inaccuracy in the actual year of the diagnosis of asthma, in addition to the unknown duration of symptoms before the diagnosis. In addition, some inaccuracy in the year of diagnosis of farmer's lung may occur due to the possibility of a delayed diagnosis or delayed registration.

A special refund is also provided for obstructive pulmonary diseases other than asthma if there is significant bronchodilator responsiveness. The proportion of smokers among farmer's lung patients is low $(3,7,12)$. The mean age and gender distribution of farmer's lung patients in this study were close to those of 2 other Finnish follow-up studies $(3,7)$. Thus the smoking habits of the patients can be assumed to be similar to those of the follow-up studies. The number of patients with chronic bronchitis and emphysema and also entitlement to a special refund for asthma medication was likely to be low in our study.

In conclusion, data concerning the entitlement to receive a special refund for asthma medication indicated that asthma was present in $1 \%$ of the patients in the year preceding the diagnosis of farmer's lung. In the year of the diagnosis of farmer's lung and during the 5 follow-up years, asthma was diagnosed in an additional $6 \%$ of the patients. In our study the prevalence of asthma was low before the onset of farmer's lung and there was an increased incidence of asthma during the first years after an episode of farmer's lung. This finding confirms previous findings with smaller groups of patients $(3-5)$.

\section{Acknowledgments}

The authors thank Dr Jyrki Elo from the Regional Office of Social Insurance Institution in east Finland for critically reading the manuscript.

\section{References}

1. Pepys J. Hypersensitivity diseases of the lungs due to fungi and organic dusts. Monogr Allergy 1969;4:1-147.

2. Baur X, Dexheimer E. Hypersensitivity pneumonitis concomitant with acute airway obstruction after exposure to hay dust. Respiration 1984;46:354-61.

3. Kokkarinen JI, Tukiainen HO, Terho EO. Recovery of pulmonary function in farmer's lung: a five-year follow-up study. Am Rev Respir Dis 1993;147:793-6.

4. Pepys J. Hypersensitivity to inhaled organic antigens. J R Coll Physicians Lond 1967;2:42-8.

5. Cuthbert OD, Gordon MF. Ten year follow up of farmers with farmer's lung. Br J Ind Med 1983;40:173-6.

6. Terho EO. Diagnostic criteria for farmer's lung disease. Am J Ind Med 1986;10:329.

7. Mönkäre S, Haahtela T. Farmer's lung - a 5 year follow-up of eighty-six patients. Clin Allergy 1987;17:143-51.

8. Karr RM, Kohler PF, Salvaggio JE. Hypersensitivity pneumonitis and extrinsic asthma: an unusual association. Chest 1978; 74:98-102.

9. Terho EO. Work-related respiratory diseases among Finnish farmers. Am J Ind Med 1990;18:567—9.

10. Mönkäre S, Haahtela T, Ikonen M, Laitinen LA. Bronchial hyperreactivity to inhaled histamine in patients with farmer's lung. Lung 1981;159:145-51.

11. Pforte A, Schild U, Breyer G, Häussinger K, Ziegler-Heitbrock HWL. A role for IgE in extrinsic allergic alveolitis? Clin Invest 1992;70:277-82.

12. Warren CPW. Extrinsic allergic alveolitis; a disease commoner in non-smokers. Thorax 1977;32:567-9.

Received for publication: 15 April 1996 